\title{
Two-flux method for transient radiative transfer in a semitransparent layer
}

\author{
ROBERT SIEGEL \\ NASA Lewis Research Center. Cleveland, OH 44135. U.S.A.
}

(Receired 8 December 1994 and in final form 9 Mal 1995)

\section{INTRODUCTION}

In semitransparent materials where thermal radiation can affect internal temperature distributions. transient behavior has been studied much less than steady-state. To obtain transient solutions, numerical procedures such as finite difference and finite element methods have been used to solve the radiative transfer relations coupled with the transient energy equation. Some of the literature has been reviewed in [1-3]. In [3] transient solutions were obtained for a layer with a refractive index larger than one with external convection and radiation at each boundary; these results using the exact equations of radiative transfer will be used for comparison with the present two-flux calculations.

Various multi-flux methods have been discussed [4] as a simplification for computing the radiative flux term in the energy equation. For the general boundary conditions of external convection and radiation on a layer with diffuse interfaces it was shown in [5] that the two-flux method can be used to predict accurate steady-state temperature distributions and heat fluxes. The purpose of this note is to show that the two-flux method can be used to obtain transient solutions in materials with large refractive indices that are typical of ceramics. Good predictions of transient temperature distributions are obtained as verified by comparison with implicit numerical solutions from [3] where the exact radiative transfer equations were used. The information on transient behavior is of interest for computing heat transfer performance in ceramics being developed for high temperature use in advanced aircraft engines.

An advantage of the two-flux method is that isotropic scattering is included without any additional complication. Some transient results with large scattering are given to illustrate scattering effects: the solutions in [3] are for absorption only.

\section{ANALYSIS}

Energy and wio-fux equations

A plane layer of thickness $D$. Fig. 1 , is a heat conducting. gray emitting. absorbing, and isotropically scattering medium with $n \geqslant 1$, and its boundaries are assumed diffuse. The laver is initially at uniform temperature $T_{1}$ and is placed in surroundings so each boundary receives radiative energy and is subject to convection. Transient temperature distributions are to be obtained in the layer until steady-state is reached corresponding to the external radiation and convection conditions.

The transient energy equation in dimensionless form is [3]

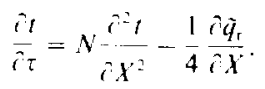

Properties are assumed independent of temperature. The gradient of the radiative flux. $\rho_{r}(X, \tau) / \gamma X$, is obtained from the two-flux relation using the Milne Eddington approximation $[4,6]$,

$$
\left.\frac{i \tilde{q}_{1}(X, \tau)}{\partial X}=n_{1}\right)(1-\Omega)\left[4 n^{2} t^{4}(X, \tau)-\tilde{G(X, \tau)]}\right.
$$

where $\tilde{G}(X, \tau)$ is related to $\hat{q}_{\mathrm{r}}(X, \tau)$ by the equation,

$$
\frac{\lambda \tilde{G}(X, \tau)}{r X}=-3 \kappa_{1}, \tilde{q}_{r}(X, \tau)
$$

The $\tilde{q}_{\mathrm{s}}$ and $(\tilde{\xi}$ are related to the positive and negative radiative fluxes shown in Fig. 1 by $\tilde{q}_{\mathrm{r}}(X, \tau)=\tilde{q}_{\mathrm{r}}{ }^{\prime}(X, \tau)-\tilde{q}_{\mathrm{r}}(X, \tau)$ and $\tilde{G}(X, \tau)=2\left[\tilde{q}_{\mathrm{r}}^{\prime}(X, \tau)+\tilde{q}_{\mathrm{r}}(X, \tau)\right]$.

Bondary and initial conditions.

The convective boundary conditions on the sides of the laver are

$$
\left.\partial \frac{\partial}{\partial X}\right|_{x=}=-\frac{I_{1}}{4 N}\left[t_{y 1}-l(0, \tau)\right]
$$

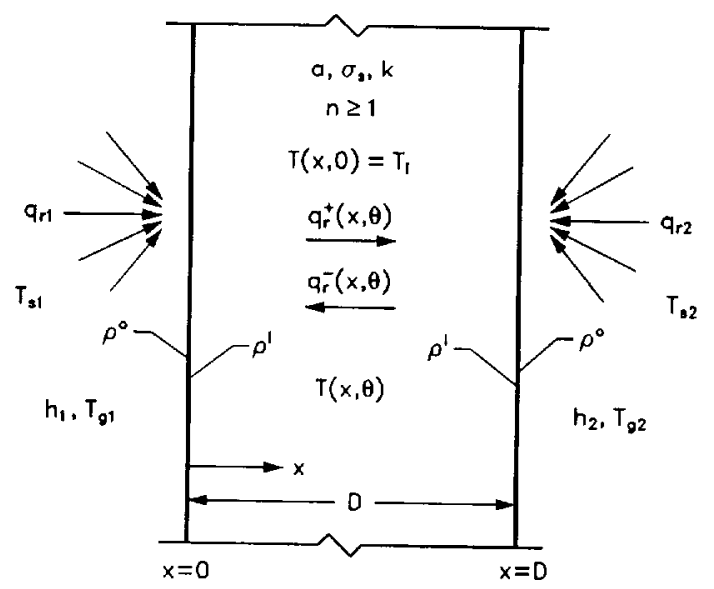

Fig. 1. Geometry and nomenclature for Iransient radiation and conduction in a semitransparent layer with isotropic scattering. 


\section{NOMENCLATURE}

a absorption coefticient in layer [m ']

$B \quad$ the quantity $3 \kappa_{\bar{D}}(1-\Omega)$

C specific heal of radiating medium

[J kg $\left.{ }^{-1} \mathrm{~K}^{-1}\right]$

D thickness of semitransparent lityer $[\mathrm{m}]$

$G$ the flux quantity $2\left(q_{\mathrm{r}}{ }^{\prime}+q_{\mathrm{t}}\right)[\mathrm{W} \mathrm{m}]$; $\tilde{G}=G \sigma T^{\downarrow}$

$h_{1}, h_{2}$ convective heat transfer coefficients al $x=0$ and $D$ [ $\mathrm{W} \mathrm{m}^{-2} \mathrm{~K}$ ']

$H_{1}, H_{2}$ dimensionless parameters. $h_{1} \sigma T_{\mathrm{i}}^{3}$ and $h_{2} \sigma \sigma T_{i}^{3}$

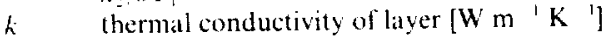

$n \quad$ refractive index of layer

$N \quad$ conduction radiation parameter, $k / 4 \sigma T_{i}^{3} D$

$q$ radiative flux in the $x$-direction [W $m{ }^{\prime}$ ]; $j_{i}=q \sigma T^{+}$

$4_{1} \cdot \psi_{2}$, external radiation fuxes incident at $x=0$ and $D[\mathrm{~W} \mathrm{~m}-1$

$\bar{q}_{1+1}, \bar{y}_{12}$ dimensionless radiation fluxes. $q_{1}: \sigma T_{1}^{4}, q_{1}: \sigma T_{1}^{+}$

T absolute lemperatture $[\mathrm{K}]: t=T T$
$T_{\mathrm{K} 11}, T_{\mathrm{k} 2}$ gas temperatures for convection at $\mathrm{x}=0$ and $D[\mathrm{~K}]$

$t_{\mathrm{F} 1}, t_{\mathrm{g} 2}$ dimensionless gas temperatures, $T_{\mathrm{k} 1} T_{1}$. $T_{\mathrm{z} z}: T_{\mathrm{i}}$

$T . \quad$ initial unilorm temperature of layer (used as a reference $T)[\mathrm{K}]$

$x$ coordinate in direction across layer $[\mathrm{m}]$ : $X=x D$.

\section{Greek symbols}

0 time [s]

$\kappa_{\mathrm{I}} \quad$ optical thickness of layer, $(a+\sigma$,

$\mu$ density of semitransparent medium [ $\mathrm{kg} \mathrm{m}$ "]

$\rho^{\prime}, \rho^{\prime \prime} \quad$ internal and external reflectivities at a boundary

$\sigma \quad$ Stefan Boltzmann constant $\left[W m^{2} \mathbf{K}{ }^{4}\right]$

$\sigma$. scattering coefficient in layer $\left[\mathrm{m}^{\prime}\right]$

$\tau$ dimensionless time. $\left(4 \sigma T_{i}^{3} / \rho c D\right) 0$

$\Omega \quad$ seattering albedo, $\sigma,\left(a+\sigma_{s}\right)$.

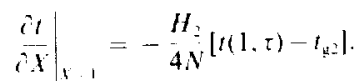

The radiative boundary conditions must now be specified including the effects of internal and external reflections at the surfaces. By considering the incident and reflected fluxes it an interface the following boundary relations between $(\bar{r}$ and $d^{2}$ were developed at each boundary [5]

$$
\begin{aligned}
& \tilde{G}(0 . \tau)=4 \frac{1-\rho^{\prime \prime}}{1-\rho^{\prime}} \tilde{q}_{11}-2 \frac{1+\rho^{\prime}}{1} \tilde{q}_{1}(0, \tau) \\
& \widetilde{G}(1, \tau)=4 \frac{1-\rho^{\prime \prime}}{1-\rho^{\prime}} \bar{q}_{12}+2 \frac{1+\rho^{\prime}}{1-\rho^{\prime}} \dot{q}_{1}(1, \tau) .
\end{aligned}
$$

To begin the (ransient solution of equation (1) the specified initial condition is a uniform temperature $T(x, 0)=T_{\text {i }}$ so $t(X, 0)=1$. Initial distributions are also needed for $\bar{q}_{t}(X, 0)$ and $\tilde{G}(X, 0)$. By differentiation. equations $(2)$ and (3) can be combined to eliminate cither $\phi_{t}(X, \tau)$ or $(\tilde{G}(X, \tau)$ to give a second order equation for either of these quantities. With $t=1$ initially, these equations are solved analytically to give.

$$
\begin{aligned}
& \vec{G}(X, 0)=C_{1} \mathrm{e}^{\bar{B}}+C_{2} \mathrm{c}^{2}{ }^{a x}+4 n^{2} \\
& \tilde{q}_{1}(X, 0)=\frac{\vec{B}}{3_{\left.\mathrm{H}_{1}\right)}}\left(C_{1} \mathrm{e}^{B M} \cdot C_{2} \mathrm{e}^{-{ }^{B I}}\right)
\end{aligned}
$$

where $B=3 \kappa_{i}(1-\Omega)$. $C_{1}$ and $C_{z}$ are integration constants that are obtained by applying the boundary conditions (5a) and $(5 b)$ to equation (6al). The following quantities are detined

$$
\begin{aligned}
& \alpha \equiv 1-2 \begin{array}{l}
1+\rho^{\prime} \\
1-\rho^{3}
\end{array} \\
& \beta \equiv 1+2 \begin{array}{l}
1+\mu^{\prime} \\
1-\rho^{\prime}
\end{array} 3
\end{aligned}
$$

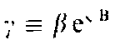

$$
\begin{aligned}
& \delta \equiv x \mathrm{e}^{\mathrm{B}} \\
& S_{1} \equiv-4 n^{2}+4 \frac{1-\rho^{\prime \prime}}{1-\rho^{\prime}} \dot{q}_{r}
\end{aligned}
$$

$$
S_{2} \equiv-4 n^{2}+4 \frac{1-p^{\prime \prime}}{1-\rho^{2}} \tilde{q}_{r_{2}} \cdot
$$

Then the integration constants are,

$$
\begin{aligned}
& C_{1}=\frac{-\beta S_{2}+\delta S_{1}}{-\beta_{i}+x \delta} \\
& C_{2}=\frac{\alpha S_{2}-\gamma S_{1}}{-\beta_{i}+x \delta} .
\end{aligned}
$$

Numerical solution

Starting with the initial $t(X, 0)$ and $\tilde{q}_{\mathrm{r}}(X, 0)$ relations, equation (1) was integrated forward in time using the following explicit finite-difference algorithm at the interior grid points

$$
\begin{aligned}
t(X, \tau+\Delta \tau)= & l(X, \tau)+\frac{N \Delta \tau}{(\Delta X)^{2}}[\imath(X+\Delta X, \tau) \\
& -2 t(X, \tau)+t(X-\Delta X, \tau)]-\left.\frac{\Delta \tau}{4} \frac{\tau \dot{q}_{1}}{\partial X}\right|_{x, \tau}
\end{aligned}
$$

The $f(\tau+\Delta \tau)$ at the boundaries were then evaluated using equations (4a) and (4b) with a three-point difference approximation for the temperature derivative

$\tau(0, \tau+\Delta \tau)$

$$
=\frac{\frac{H_{1} \Delta X}{2 N} t_{l 1}+4 /(\Delta X, \tau+\Delta \tau)-t(2 \Delta X, \tau+\Delta \tau)}{3+\frac{H_{1} \Delta X}{2 N}}
$$

$(1, \tau+\Delta \tau)$

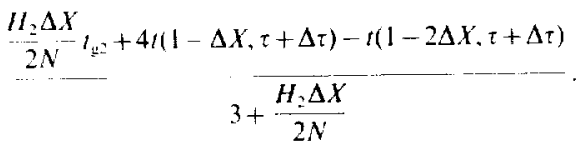

After advancing $t(X)$ each time increment the radiant flux gradient in the last term in equation (9) must be advanced to $\tau+\Delta \tau$. This was done by solving equations (2) and (3) simultaneously for $\tilde{G}(X, \tau+\Delta \tau)$ and $\tilde{q}_{1}(X, \tau+\Delta \tau)$ using $f(X, \tau+\Delta \tau)$ on the right-hand side of equation (2). Then $\bar{g}_{\mathrm{r}}(X, \tau+\Delta \tau) \subset X$ was evaluated from equation (2). The sim- 
ultaneous solution of equations (2) and (3) was carried out using a fourth-order Runge-Kutta method with a shooting procedure to satisfy the boundary conditions at $X=0$ and 1. To begin the solution the value of $\bar{q}_{\mathrm{r}}(X=0)$ from the previous time step was used as an estimate, and the boundary condition equation (5a) was solved for $\tilde{G}(X=0)$. The solution was then carried out by Runge - Kutta integration from $X=0$ to $X=1$. The values of $\tilde{q}_{\mathrm{r}}$ and $\tilde{G}$ obtained at $X=1$ were checked to see if they satisfy the boundary condition in equation (5b). An iteration was performed on $\tilde{q}_{r}(X=0)$ until equation (5b) was satisfied; the type of iterative method used is described in [7]. The shooting method used here is convenient for absorption optical thicknesses, $a D \leqslant 8$. This two-point boundary value solution method becomes difficult when there is a large $a D$ that causes the conditions at the two boundaries to become less directly related. It is possible (a)

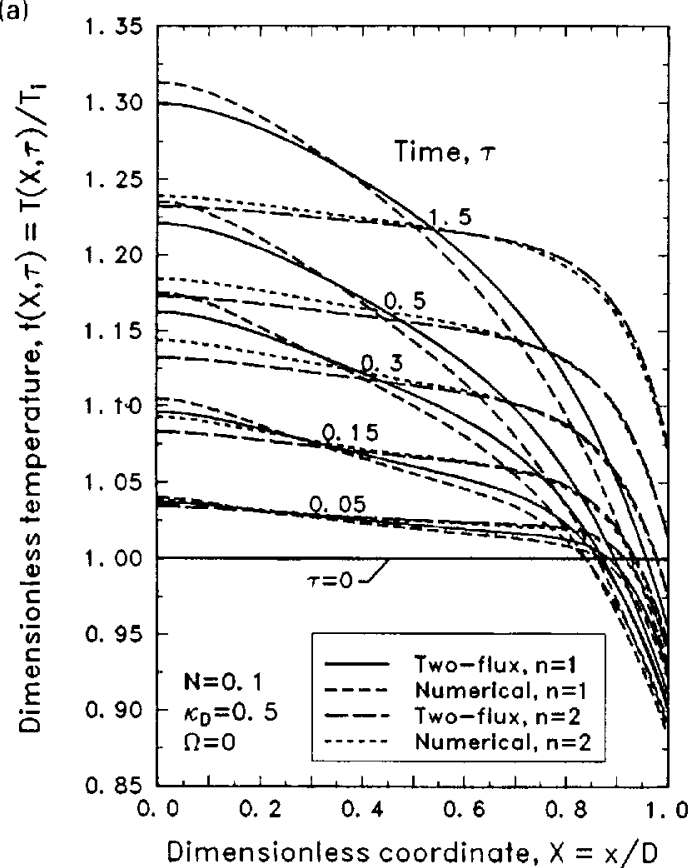

(b)

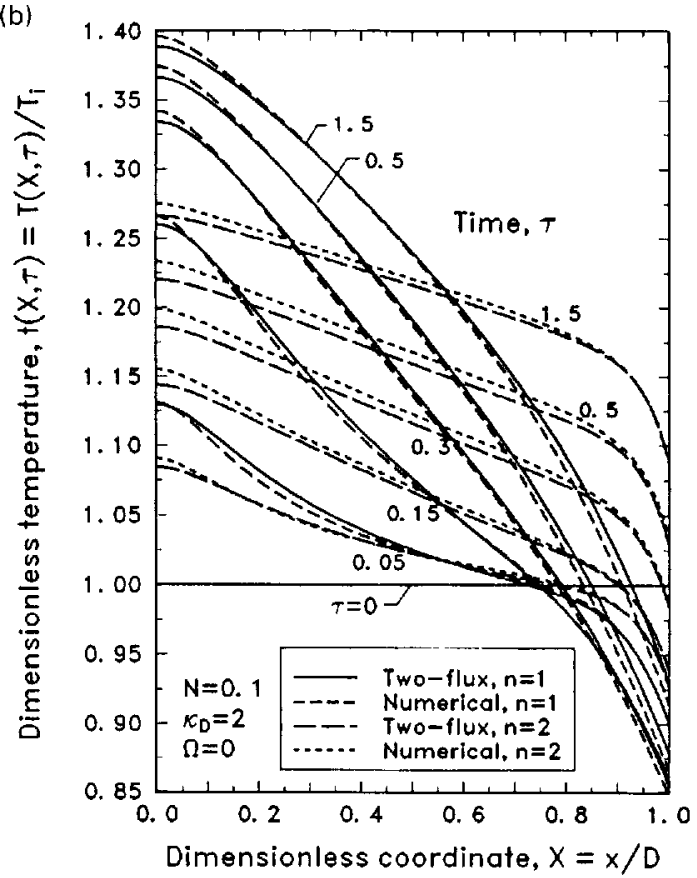

(c)

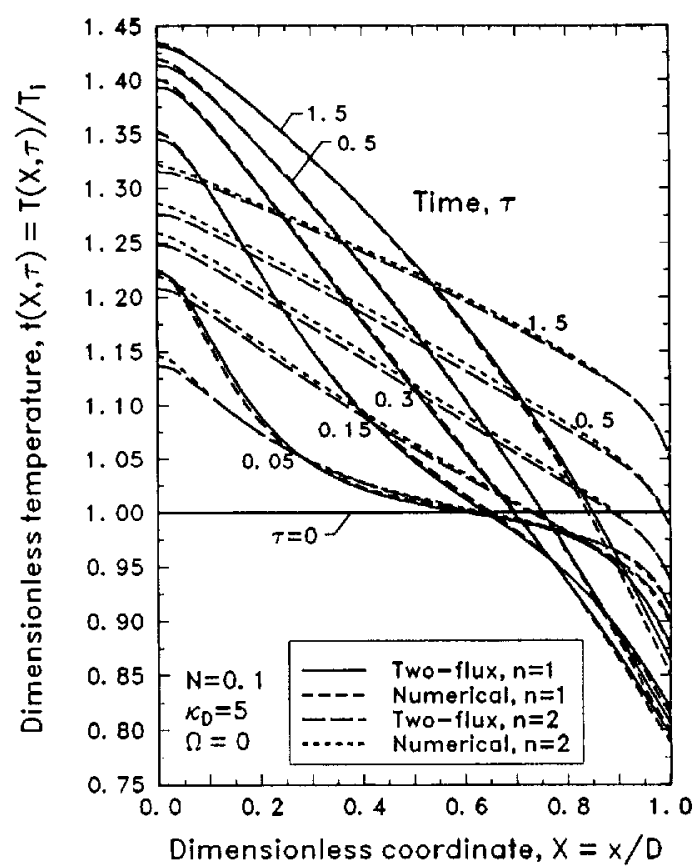

Fig. 2. Two-flux and exact results for transient temperature distributions in a layer initially at uniform temperature after exposure to radiation on one side and convective cooling on the other side: no scattering. $\Omega=0$. Parameters: $N=0.1, \tilde{q}_{t 1}=1.5^{4}, \tilde{q}_{t 2}=0.5^{4}, H_{1}=0 . H_{2}=1, t_{g-2}=0.5$. (a) Optical thickness. $k_{12}=0.5 ;$ (b) optical thickness, $k_{1}=2 ;$ (c) optical thickness, $k_{1}=5$. 
that other numerical techniques could partially climinate this difficulty: a method using a Green's function is presently being developed. Using the $f(X)$ and $\tilde{G}(X)$ the radiant flux gradient was evaluated from equation (2). The temperature distribution was then advanced to the next time increment using equations (9) and (10).

After checking various grid sizes it was found that 41 evenly spaced points across the layer gave accurate solutions. Corresponding to this grid size $(\Delta X=0.025)$ and for $N=0.1$, is used for the results given here, the $\Delta \tau$ for at stable explicit calculation was estimated from the criterion for solving the transient heat conduction equation. The value $\Delta \tau=0.0025$ provided stable solutions for all of the results calculated here.

\section{RESULTS AND DISCUSSION}

The transient temperature distributions given here start from a uniform initial temperature $T(X, 0)=T$, so that $I(X, 0)=1$. Figure 2 shows typical comparisons of two-flux results for $l(X, \tau)$ with those from [3] using an implicit finitedifference method, and the exact equation of transfer to evaluate the radiative flux gradient in the energy equation The laver is heated on the hot side $(X=0)$ by a radiative flux cqual to that from black body surroundings at $T_{\mathrm{v}}=1.5 T_{1}$; at $\mu=1, T_{3}=0.5 T$, so there is a net radiative cooling at that side. These illustrative results examine the thermal behavior of a layer that is convectively cooled only on the side away from where the radiative heating occurs. This simulates possible conditions for the wall of a combustion chamber where there is radiative heating from combustion galses on one side. and that side is not being film cooled.

The three parts of Fig. 2 are for optical thicknesses of 0.5 2 and 5 with no scattering. For $\left.k_{1}\right)=0.5$ the layer is somewhat optically thin. For $\kappa_{12}=2$ the optical thickness is such that maximum internal radiative effects are expected : for $h_{1}=5$ the layer is somewhat optically thick. Each part of the figure shows results for $n=1$ and 2. The two-flux curves are solid or long dashes; the numerical results using the exact transfer equations are medium or short dashes. When $\tau=1.5$ the temperatures are within $1 \%$ of steady state. At $X=0$ the temperature profiles have a zero derivative from the absence of convective cooling at that boundary. The convective cooling at $f=1$ produces al ratpid temperature decrease near that boundary

The results using the two-flux method agree within reasonable error with predictions using the exact transfer equations. The largest deviations, which are for $k_{1},=0.5$. are only a few per cent, and agreement is much better for $k_{1}=5$. As $n$ increases, internal reflections make the temperature distributions more uniform. In most instances agreement of the (wo-flux results wats a little betler for $n=1$ than for $n=2$.

The effect of scattering is illustrated in Fig. 3 for $n=1$ and 2 . The optical thickness is constant. $k_{12}=5$. so an increase in scattering corresponds to a decrease in absorption. The result is that the transient temperatures are decreased with increasing $\Omega$. For $n=2$ in Fig. 3 (b) the temperatures are somewhat more uniform than for $n=1$ in Fig. 3(a) [note that the ordinate scales are different in Figs. $3(a)$ and $3(b)]$. Compared with Fig. 3(a), increasing $\Omega$ in Fig. 3(b) does not have as large an effect in reducing the temperatures. For $n=2$ the layer has internal reflections that make scattering more effective in augmenting absorption. For $\Omega=0.99$ this makes the temperatures larger for $n=2$ than for $n=1$.

\section{CONCLUSIONS}

The two-flux method was used to obtain transient solutions for a plane layer including internal reflections and scattering. The layer was initially at uniform temperature, and was heated or cooled by external radiation and convection. The two-fiux equations were examined as a means for eva- luating the radiative flux gradient in the transient energy equation. Comparisons of transient temperature distributions using the two-flux method were made with results where the radiative flux gradient was evaluated from the exact radiative transfer equations. Good agreement was obtained for optical thicknesses from 0.5 to 5 and for refractive indices of 1 and 2. Illustrative results obtained with the two-flux method demonstrate the effect of isotropic scat-
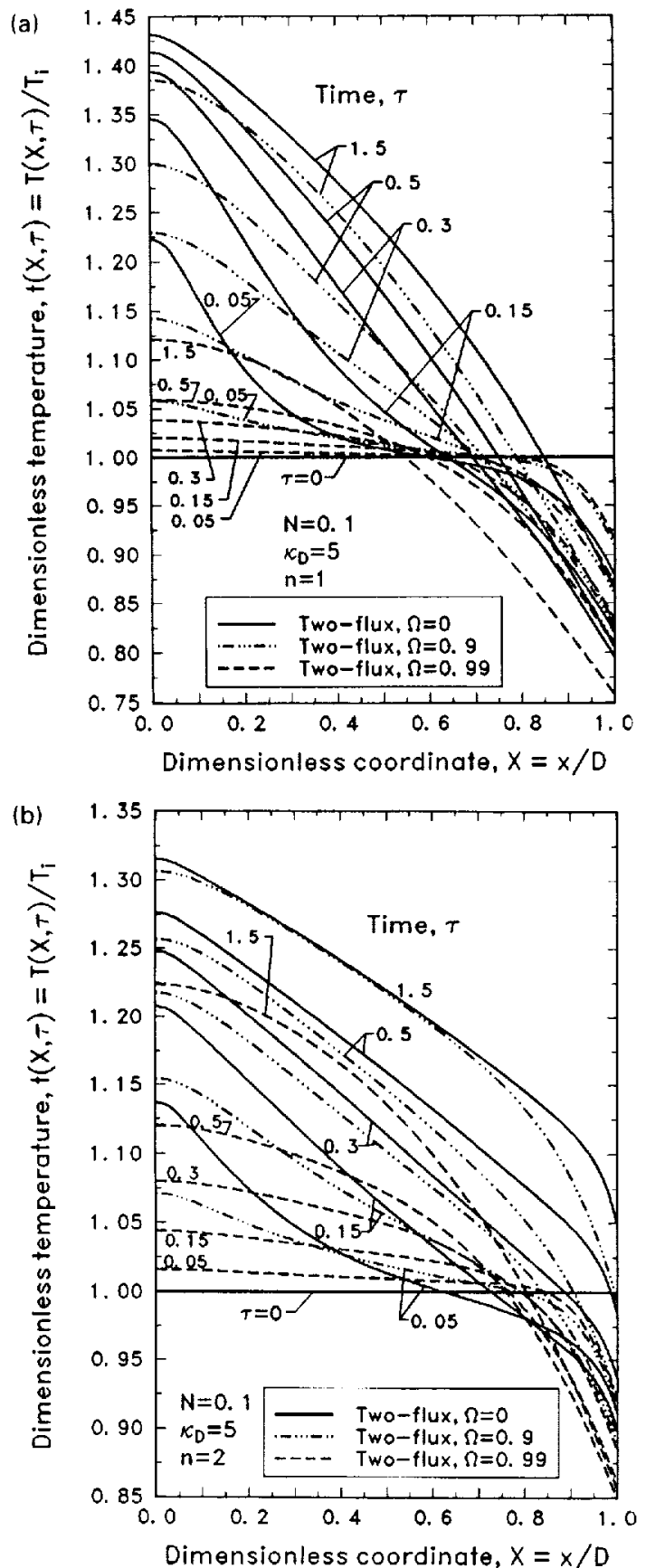

Fig. 3. Effect of scattering on temperature distributions in a layer initially at uniform temperature after exposure to radiation on one side and convective cooling on the other side. Parameters: $\kappa_{1}=5, N=0.1, \tilde{q}_{\mathrm{s}}=1.5^{4}, \tilde{q}_{\mathrm{r} 2}=0.5^{4}$, $H_{1}=0, H_{2}=1, t_{\mathrm{g} 2}=0.5$. (a) Refractive index, $n=1$ : (h) refractive index. $n=2$. 
tering coupled with changing the refractive index. For small absorption with large seattering the maximum layer temperature is increased when the refractive index is increased. For larger absorption the effect is opposite. and the maximum temperature decreases with increased refractive index.

\section{REFERENCES}

1. C.-Y, Wu and N.-R, Ou. Transient two-dimensional radiative and conductive heat transfer in a scattering medium. Int. J. Heat Mass Transfer 37, 26752686 (1994).

2. C. C. Lii and M. N. Ozzisik, Transient radiation and conduction in an absorbing. emitting. scattering slab with reflective boundaries, Int. J. Heat Mass Transfer 15, 1175 1179 (1972).

3. R. Siegel, Transient heat transfer in a semitransparent radiating layer with boundary convection and surface reflections, Int. J. Heat Mass Transfer 39, 69.79 (1996).

4. R. G. Siddall, Flux methods for the analysis of radian heat transfer. Procesdings of the Fourth Simposium on Flames and Industri. Imperial College. London, U.K Paper 16. pp. 169 -179. The Institute of Fuel (1972)

5. R. Siegel and C. M. Spuckler, Approximate solution methods for spectral radiative transfer in high refractive index layers, $\mathrm{lm}$. J. Heat Mass Transfer 37 (Suppl. 1). 40.3413 (1994).

6. R. Siegel and J. R. Howell, Thermal Radiation Heat Tramsfer (3rd Edn), pp. 772 773. Hemisphere, Washington D.C. (1992)

7. W. H. Press, B. P. Flannery. S. A. Teukolsky and W. T Vetterling. Numerical Recipes (FORTRAN Version), pp. 582 585. Cambridge University Press. Cambridge, U.K. (1989). 
. 